\title{
Exotic hadrons from BESIII
}

\author{
Chang-Zheng Yuan ${ }^{1,2, *}$ \\ (For the BESIII Collaboration) \\ ${ }^{1}$ Institute of High Energy Physics, Chinese Academy of Sciences, Beijing, China \\ ${ }^{2}$ University of Chinese Academy of Sciences, Beijing, China
}

\begin{abstract}
We report the progress in the study of the exotic hadrons, also called $\mathrm{XYZ}$ states, at BESIII experiment with its unique data samples in $e^{+} e^{-}$annihilation at center-of-mass energies of 3.8-4.6 GeV. The talk covers the observation of the $Z_{c}(3900)$ and $Z_{c}(4020)$ and new measurements related to them; updated measurement of the resonant parameters of the $Y(4260)$ in $e^{+} e^{-} \rightarrow \pi^{+} \pi^{-} J / \psi$ and discovery of its new decay modes $\pi^{+} \pi^{-} \psi(2 S), \omega \chi_{c 0}, \pi^{+} \pi^{-} h_{c}$, and $D^{0} D^{*-} \pi^{+}+c . c$.; and observation of $X(3872)$ in $e^{+} e^{-}$annihilation.
\end{abstract}

\section{Introduction}

In the conventional quark model, mesons are composed of one quark and one anti-quark, while baryons are composed of three quarks. Although this picture is very simple, it describes almost all the hadrons observed so far [1]. However, many charmonium-like states were discovered at two $B$-factories BaBar and Belle in the first decade of this century [2]. Whereas some of these are good candidates of charmonium states, many other states have exotic properties, which may indicate that exotic states, such as multi-quark state, hadronic molecule, or hybrid, have been observed [3]. Experimentally, these states are also called $\mathrm{XYZ}$ states, to indicate their nature is still unclear.

BaBar and Belle experiments finished their data taking in 2008 and 2010, respectively, and the data are still used for various physics analyses. In 2008, the BESIII [4], a $\tau$-charm factory experiment at the BEPCII $e^{+} e^{-}$collider, started data taking, and contributed to the study of the XYZ particles ever since. In this article, we focus on the measurements of the three mostly studied XYZ states, i.e., the $X(3872)$, the $Y(4260)$, and the $Z_{c}(3900)$, at BESIII [5].

\section{The charged charmonium-like state $Z_{c}(3900)$}

\subsection{Discovery of the $Z_{c}(3900)$}

The BESIII experiment studied the $e^{+} e^{-} \rightarrow \pi^{+} \pi^{-} J / \psi$ process at a center-of-mass (c.m.) energy of $4.26 \mathrm{GeV}$ using a $525 \mathrm{pb}^{-1}$ data sample [6], with $J / \psi$ decays into a pair of $e^{+} e^{-}$or

\footnotetext{
*e-mail: yuancz@ihep.ac.cn
} 

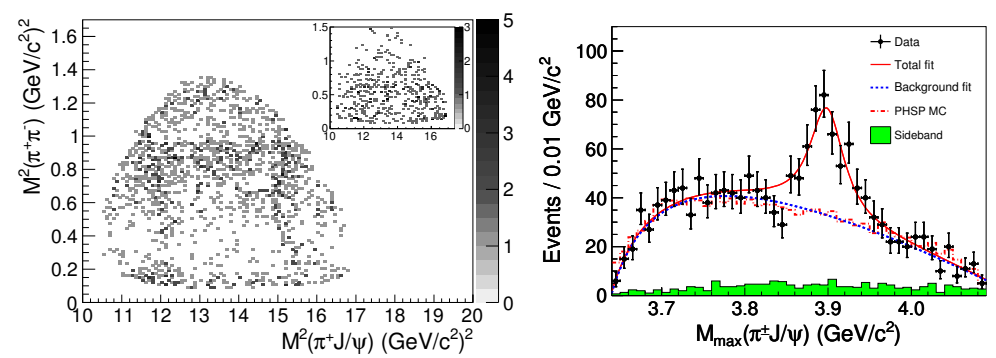

Figure 1. Dalitz plot for selected $e^{+} e^{-} \rightarrow \pi^{+} \pi^{-} J / \psi$ events in the $J / \psi$ signal region (left panel), and unbinned maximum likelihood fit to the distribution of the $M_{\max }(\pi J / \psi)$ (right panel).

$\mu^{+} \mu^{-}$. The $J / \psi$ signal is selected by requiring the invariant mass of the lepton pair is consistent with the $J / \psi$, and a sample of $1595 \pi^{+} \pi^{-} J / \psi$ events with a purity of $90 \%$ is obtained. The intermediate states are studied by examining the Dalitz plot (shown in Fig. 1) of the selected candidate events.

A structure at around $3.9 \mathrm{GeV} / c^{2}$ was observed in the $\pi^{ \pm} J / \psi$ invariant mass distribution with a statistical significance larger than $8 \sigma$, which is referred to as the $Z_{c}(3900)$. A fit to the $\pi^{ \pm} J / \psi$ invariant mass spectrum with a constant width Breit-Wigner (BW) function (Fig. 1), neglecting interference with other amplitudes, results in a mass of $(3899.0 \pm 3.6 \pm 4.9) \mathrm{MeV} / c^{2}$ and a width of $(46 \pm 10 \pm 20) \mathrm{MeV}$. The $Z_{c}$ (3900) state was reported shortly after at Belle [7] with initial state radiation (ISR) data and with CLEO-c data at a c.m. energy of $4.17 \mathrm{GeV}$ [8], and the mass and width agreed very well with the BESIII measurement.

\subsection{Spin-parity of the $Z_{c}(3900)$}

BESIII determines the spin-parity of the $Z_{c}$ (3900) based on a partial wave analysis (PWA) of $e^{+} e^{-} \rightarrow \pi^{+} \pi^{-} J / \psi$ events at $\sqrt{s}=4.23$ and $4.26 \mathrm{GeV}$ [9]. Following the event selection reported in Ref. [6], the numbers of selected candidate events are 4154 at $\sqrt{s}=4.23 \mathrm{GeV}$ and 2447 at $\sqrt{s}=4.26 \mathrm{GeV}$, with 365 and 272 background events, respectively, estimated by using the $J / \psi$ mass sidebands.

The fit indicates that the spin-parity $J^{P}=1^{+}$of the $Z_{c}(3900)$ are favored by more than $7 \sigma$ over other quantum numbers $\left(0^{-}, 1^{-}, 2^{-}\right.$, and $\left.2^{+}\right)$. The pole mass of the $Z_{c}(3900)$ is measured as $(3881.2 \pm 4.2 \pm 52.7) \mathrm{MeV} / c^{2}$ and pole width $(51.8 \pm 4.6 \pm 36.0) \mathrm{MeV}$. The Born cross sections for $e^{+} e^{-} \rightarrow \pi^{+} Z_{c}(3900)^{-}+$c.c. $\rightarrow \pi^{+} \pi^{-} J / \psi$ are measured to be $(21.8 \pm 1.0 \pm 4.4) \mathrm{pb}$ at $\sqrt{s}=4.23 \mathrm{GeV}$ and $(11.0 \pm 1.2 \pm 5.4)$ pb at $\sqrt{s}=4.26 \mathrm{GeV}$.

\subsection{Observation of $Z_{c}(3900)^{\mp} \rightarrow\left(D \bar{D}^{*}\right)^{\mp}$}

With the data sample at $\sqrt{s}=4.26 \mathrm{GeV}$, the BESIII experiment studied $e^{+} e^{-} \rightarrow \pi^{ \pm}\left(D \bar{D}^{*}\right)^{\mp}$ with single-tag method (only one of the two $D$ mesons is reconstructed) and observed the open-charm decay $Z_{c}(3900)^{ \pm} \rightarrow\left(D \bar{D}^{*}+c . c .\right)^{ \pm}[10]$. The analysis is refined with double-tag method (both $D$ mesons are reconstructed) and more luminosity [11]. In this analysis, both $e^{+} e^{-} \rightarrow \pi^{+} D^{0} D^{*-}+$ c.c. and $\pi^{+} D^{-} D^{* 0}+$ c.c. are measured with data samples at $\sqrt{s}=4.23$ and $4.26 \mathrm{GeV}$. The double $D$ tag technique allows the use of more $D$ decay modes and the background level is greatly suppressed. 
The double-tag analysis only has $\sim 9 \%$ events in common with the single-tag analysis, so the two analyses are almost statistically independent and the results can be combined to have a better measurement. The combined pole mass and width are $(3882.2 \pm 1.1 \pm 1.5) \mathrm{MeV} / \mathrm{c}^{2}$ and $(26.5 \pm 1.7 \pm 2.1) \mathrm{MeV}$, respectively. The combined production rate $\sigma\left(e^{+} e^{-} \rightarrow \pi^{\mp} Z_{c}(3900)^{ \pm}\right) \times$ $\mathcal{B}\left(Z_{c}(3900)^{ \pm} \rightarrow\left(D D^{*}\right)^{ \pm}\right)$is $(104.4 \pm 4.8 \pm 8.4) \mathrm{pb}$ at $\sqrt{s}=4.26 \mathrm{GeV}$. The production rate $\sigma\left(e^{+} e^{-} \rightarrow \pi^{\mp} Z_{c}(3900)^{ \pm}\right) \times \mathcal{B}\left(Z_{c}(3900)^{ \pm} \rightarrow\left(D D^{*}\right)^{ \pm}\right)=(141.6 \pm 7.9 \pm 12.3)$ pb at $\sqrt{s}=4.23 \mathrm{GeV}$ which is from double-tag method only.

\subsection{Evidence for $Z_{c}(3900) \rightarrow \rho \eta_{c}$}

BESIII searches for $e^{+} e^{-} \rightarrow \pi^{+} \pi^{-} \pi^{0} \eta_{c}$ and intermediate states decay into $\rho \eta_{c}$ with data collected at $4.23,4.26$, and $4.36 \mathrm{GeV}$ [12]. The recoil mass of one charged pion (equivalent to the invariant mass of $\rho^{ \pm} \eta_{c}$ ) is shown in Fig. 2 for the data at $\sqrt{s}=4.23 \mathrm{GeV}$, the $Z_{c}(3900)^{ \pm}$ signal is found while there is no significant $Z_{c}(4020)^{ \pm}$signal. The $\rho^{ \pm} \eta_{c}$ invariant mass distribution is fitted with the contributions from $Z_{c}(3900)$ and $Z_{c}(4020)$ together with a smooth background. $240 \pm 56 Z_{c}(3900)^{ \pm}$events is observed with a statistical significance of $4.3 \sigma$ (3.9 $\sigma$ including the systematical uncertainty). The $Z_{c}(3900)$ signals at other c.m. energies and the $Z_{c}(4020)$ signals at all the c.m. energies are not statistically significant.
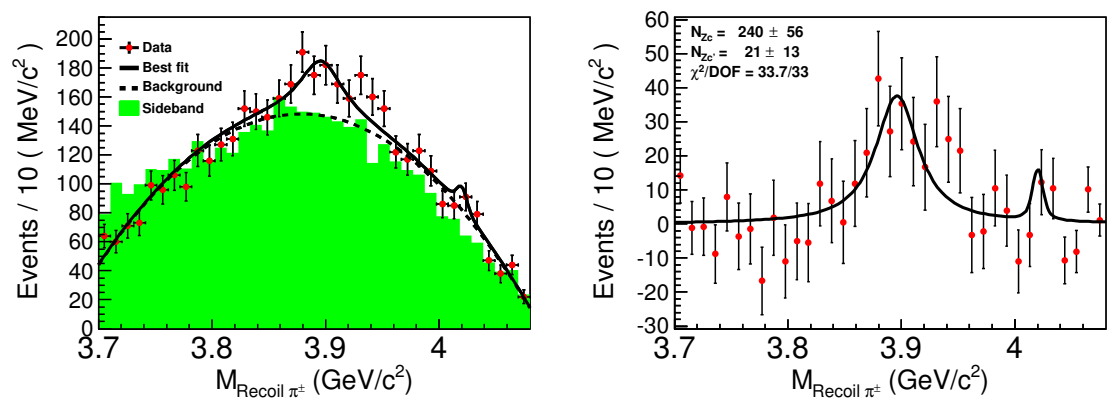

Figure 2. The $\pi^{ \pm}$recoil mass distribution in $e^{+} e^{-} \rightarrow \pi^{ \pm} \rho^{\mp} \eta_{c}$ at $\sqrt{s}=4.23 \mathrm{GeV}$ and the fit with $Z_{c}(3900 / 4020)^{ \pm}$signals (left panel); and the same plot with background subtracted (right panel). Dots with error bars are data, shaded histogram is from $\eta_{c}$ sidebands, normalized to the number of backgrounds from the fit, the solid lines are total fit and the dotted line is background.

Using the results from Ref. [9], the ratio of the branching fractions of different $Z_{c}(3900)^{ \pm}$ decays is calculated as $R_{Z_{c}(3900)}=\frac{\mathcal{B}\left(Z_{c}(3900) \rightarrow \rho \eta_{c}\right)}{\mathcal{B}\left(Z_{c}(3900) \rightarrow \pi J / \psi\right)}=2.1 \pm 0.8$ at $\sqrt{s}=4.23 \mathrm{GeV}$ and less than 6.4 at $\sqrt{s}=4.26 \mathrm{GeV}$ at the $90 \%$ C.L. The theoretical predictions for this ratio varies depending on model assumptions and ranges from a few per cent to a few hundreds [13-18].

\subsection{Observation of the $Z_{c}(4020)$}

The partner state of the $Z_{c}(3900)$ close to the $D^{*} \bar{D}^{*}$ is searched in $e^{+} e^{-} \rightarrow \pi^{+} \pi^{-} h_{c}$ and $e^{+} e^{-} \rightarrow\left(D^{*} \bar{D}^{*}\right)^{ \pm} \pi^{\mp}$. BESIII measures cross sections of $e^{+} e^{-} \rightarrow \pi^{+} \pi^{-} h_{c}$ at c.m. energies of 3.90-4.42 GeV [19]. Intermediate states are studied by examining the Dalitz plot of the selected $\pi^{+} \pi^{-} h_{c}$ candidate events. There is distinct signal for an exotic charmonium-like structure in the $\pi^{ \pm} h_{c}$ system (Fig. 3). The mass and width of the $Z_{c}(4020)$ are measured to be $(4022.9 \pm 0.8 \pm 2.7) \mathrm{MeV} / c^{2}$ and $(7.9 \pm 2.7 \pm 2.6) \mathrm{MeV}$, respectively. The statistical significance of the $Z_{c}(4020)$ signal is found to be greater than $8.9 \sigma$. 

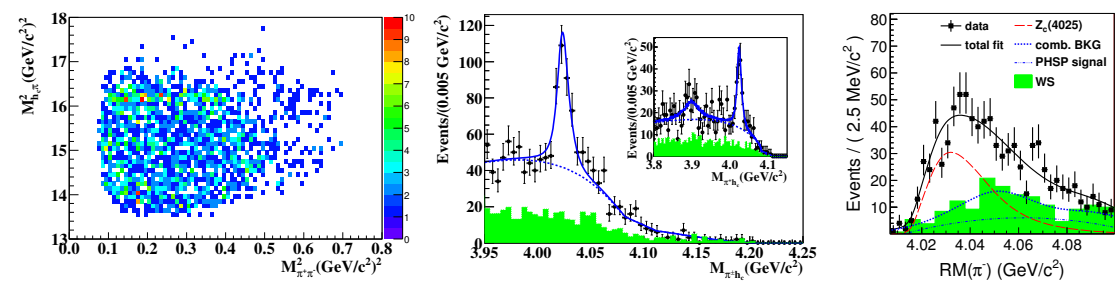

Figure 3. Dalitz plot $\left(M_{\pi^{+} h_{c}}^{2}\right.$ vs. $M_{\pi^{+} \pi^{-}}^{2}$ ) (left panel) and the fit to the $M\left(\pi^{ \pm} h_{c}\right)$ distribution (middle panel) for selected $e^{+} e^{-} \rightarrow \pi^{+} \pi^{-} h_{c}$ events, summed over all energy points; and the fit to the $\pi^{\mp}$ recoil mass spectrum (right panel) in $e^{+} e^{-} \rightarrow\left(D^{*} \bar{D}^{*}\right)^{ \pm} \pi^{\mp}$ at $\sqrt{s}=4.26 \mathrm{GeV}$.
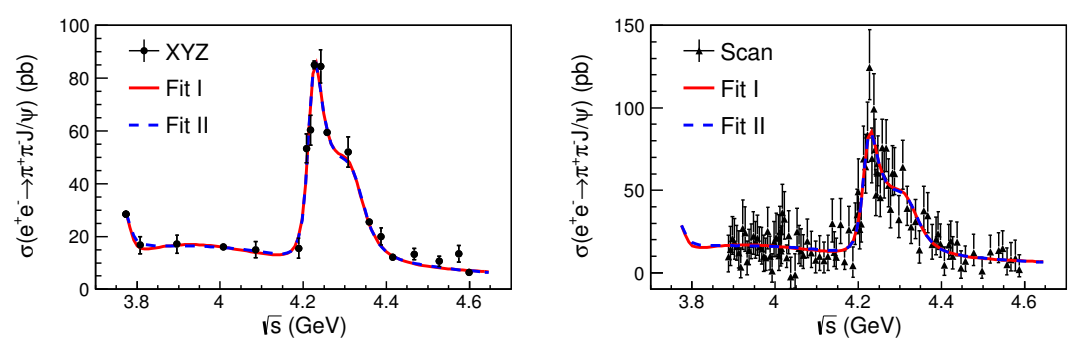

Figure 4. Measured cross section $\sigma\left(e^{+} e^{-} \rightarrow \pi^{+} \pi^{-} J / \psi\right)$ and the fit to the data.

The study of $e^{+} e^{-} \rightarrow\left(D^{*} \bar{D}^{*}\right)^{ \pm} \pi^{\mp}$ process using data at $\sqrt{s}=4.26 \mathrm{GeV}[20]$ indicates the open-charm decay $Z_{c}(4020) \rightarrow\left(D^{*} \bar{D}^{*}\right)^{ \pm}$in the $\pi^{\mp}$ recoil mass spectrum (see Fig. 3). The measured mass and width of the structure are $(4026.3 \pm 2.6 \pm 3.7) \mathrm{MeV} / c^{2}$ and $(24.8 \pm 5.6 \pm$ 7.7) $\mathrm{MeV}$, respectively, and the statistical significance is $13 \sigma$.

\section{The vector charmonium-like state $Y(4260)$}

The vector charmonium-like states with quantum numbers $J^{P C}=1^{--}$that are usually called $Y$ states, like the $Y(4260)$ [21], the $Y(4360)$ [22, 23], and the $Y(4660)$ [23]. These states show strong coupling to hidden-charm final states in contrast to the vector charmonium states in the same energy region $(\psi(4040), \psi(4160), \psi(4415))$ which couple dominantly to open-charm meson pairs [1].

\section{$3.1 e^{+} e^{-} \rightarrow \pi^{+} \pi^{-} J / \psi$}

The cross sections of $e^{+} e^{-} \rightarrow \pi^{+} \pi^{-} J / \psi$ are measured precisely at c.m. energies from 3.77 to $4.60 \mathrm{GeV}$ using $9 \mathrm{fb}^{-1}$ of BESIII data [24]. Figure 4 shows the measured cross sections, one can see clearly the $Y(4260)$ structure observed by BaBar and Belle experiments, but it is peaked at around $4.22 \mathrm{GeV}$ rather than $4.26 \mathrm{GeV}$ from the previous fits [7, 25].

Two resonant structures in the $Y(4260)$ peak region are needed in a fit to the cross sections. The first one has a mass of $(4222.0 \pm 3.1 \pm 1.4) \mathrm{MeV} / \mathrm{c}^{2}$ and a width of $(44.1 \pm 4.3 \pm 2.0) \mathrm{MeV}$, 
while the second one has a mass of $(4320.0 \pm 10.4 \pm 7.0) \mathrm{MeV} / c^{2}$ and a width of $\left(101.4_{-19.7}^{+25.3} \pm\right.$ 10.2) $\mathrm{MeV}$. The mass of first resonance is lower than that of the $Y(4260)$ and it is much narrower. The second resonance is observed in $e^{+} e^{-} \rightarrow \pi^{+} \pi^{-} J / \psi$ for the first time, with a statistical significance larger than $7.6 \sigma$.

It is worth pointing out that the lower mass structure (called $Y(4220)$ hereafter) is the main component of the $Y(4260)$ structure but with improved measurement of the resonant parameters thanks to the high luminosity data from BESIII.

$3.2 e^{+} e^{-} \rightarrow \pi^{+} \pi^{-} h_{c}$

In 2013, BESIII reported the cross section measurement of $e^{+} e^{-} \rightarrow \pi^{+} \pi^{-} h_{c}$ at $13 \mathrm{c} . \mathrm{m}$. energies from 3.9 to $4.4 \mathrm{GeV}$ [26]. In 2017, BESIII presented a follow-up study at c.m. energies from 3.9 to $4.6 \mathrm{GeV}$ [27]. The cross sections are shown in Fig. 5.
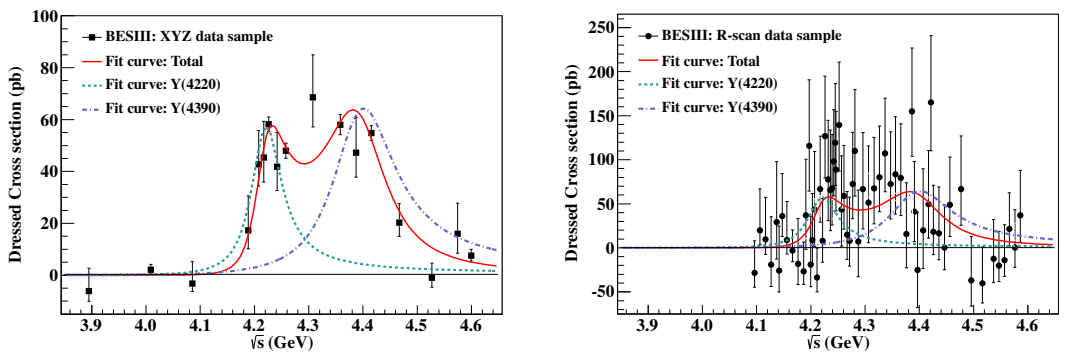

Figure 5. Fit to the cross section of $e^{+} e^{-} \rightarrow \pi^{+} \pi^{-} h_{c}$ with the coherent sum of two BW functions (solid curve).

Assuming the $\pi^{+} \pi^{-} h_{c}$ events come from two resonances, BESIII obtains $M=$ $\left(4218.4_{-4.5}^{+5.5} \pm 0.9\right) \mathrm{MeV} / c^{2}, \Gamma=\left(66.0_{-8.3}^{+12.3} \pm 0.4\right) \mathrm{MeV}$ for $Y(4220)$, and $M=\left(4391.5_{-6.8}^{+6.3} \pm\right.$ 1.0) $\mathrm{MeV} / c^{2}, \Gamma=\left(139.5_{-20.6}^{+16.2} \pm 0.6\right) \mathrm{MeV}$ for $Y(4390)$. The parameters of the low mass structure are consistent with those of the resonance observed in $e^{+} e^{-} \rightarrow \omega \chi_{c 0}$ [28] and in $e^{+} e^{-} \rightarrow \pi^{+} \pi^{-} J / \psi$ [24]. The high mass structure is different from the $Y(4360)[29,30]$ and $\psi(4415)[1]$.

\section{$3.3 e^{+} e^{-} \rightarrow \pi^{+} \pi^{-} \psi(2 S)$}

BESIII measures the cross sections of $e^{+} e^{-} \rightarrow \pi^{+} \pi^{-} \psi(2 S)$ using $5.1 \mathrm{fb}^{-1}$ of data collected from 4.0 to $4.6 \mathrm{GeV}$ [31]. The measurements are in good consistency with previous BaBar and Belle results [29, 30], but with much improved precision, as shown in Fig. 6.

As the BESIII data can only reach $4.6 \mathrm{GeV}$, the parameters of the $Y(4660)$ are fixed to Belle measurement [30] in the fit to the $e^{+} e^{-} \rightarrow \pi^{+} \pi^{-} \psi(2 S)$ cross sections. The data require a lower-mass resonance with a mass $M=(4209.5 \pm 7.4 \pm 1.4) \mathrm{MeV} / c^{2}$ and a width $\Gamma=(80.1 \pm 24.6 \pm 2.9) \mathrm{MeV}$ with a statistical significance of $5.8 \sigma$, this is the first observation of the new decay mode $Y(4220) \rightarrow \pi^{+} \pi^{-} \psi(2 S)$. The fit results are also presented in Fig. 6 .

\section{$3.4 e^{+} e^{-} \rightarrow \omega \chi_{c 0}$}

The process $e^{+} e^{-} \rightarrow \omega \chi_{c 0}$ is observed for the first time and the cross sections or the upper limits on the cross sections (when the signal is not significant) at the $90 \%$ C.L. are determined $[28,32]$. By assuming the $\omega \chi_{c 0}$ signals come from a single resonance, the mass is 

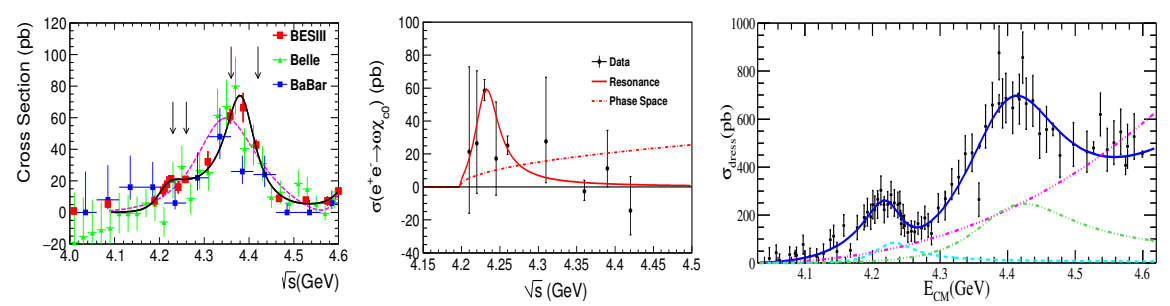

Figure 6. Cross sections of $e^{+} e^{-} \rightarrow \pi^{+} \pi^{-} \psi(2 S)$ (left panel), $\omega \chi_{c 0}$ (middle panel), and $D^{0} D^{*-} \pi^{+}+$c.c. (right panel), as a function of c.m. energy.

determined to be $(4226 \pm 8 \pm 6) \mathrm{MeV} / \mathrm{c}^{2}$, and width $(39 \pm 12 \pm 2) \mathrm{MeV}$ (shown in Fig. 6). In fact, it is in the $e^{+} e^{-} \rightarrow \omega \chi_{c 0}$ mode that the $Y(4220)$ (or $Y(4230)$ ) structure was first reported [28].

$3.5 e^{+} e^{-} \rightarrow D^{0} D^{*-} \pi^{+}+$c.c.

BESIII reported measurements of the cross section of $e^{+} e^{-} \rightarrow D^{0} D^{*-} \pi^{+}+$c.c. at c.m. energies from 4.05 to $4.60 \mathrm{GeV}$ [33] (shown in Fig. 6), which is a significant improvement over the Belle measurement [34] with ISR technique.

A fit to the cross section is performed to determine the parameters of the resonant structures. The fit yields a mass of $(4228.6 \pm 4.1 \pm 5.9) \mathrm{MeV} / c^{2}$ and a width of $(77.1 \pm 6.8 \pm 6.9) \mathrm{MeV}$ for the lower mass structure, and a mass of $(4400.1 \pm 9.3) \mathrm{MeV} / \mathrm{c}^{2}$ and a width of $(181.7 \pm 16.9) \mathrm{MeV}$ for the higher mass one. Here for the higher mass state, the errors are statistical only. This is the first observation of the $Y(4220)$ decays into open-charm final state $D^{0} D^{*-} \pi^{+}+$c.c..

\subsection{Resonant parameters of the $Y(4260)$ from combined fit}

As the cross sections of different final states have some common features, and some of the final states have been measured by different experiments, these data are used to do combined fit to extract more information about the resonant structures [35, 36].

In Ref. [35], the authors use the measured cross sections of $e^{+} e^{-} \rightarrow \omega \chi_{c 0}$ [32], $\pi^{+} \pi^{-} h_{c}$ [27], $\pi^{+} \pi^{-} J / \psi$ [24], and $D^{0} D^{*-} \pi^{+}+$c.c. [33] processes to determine the resonant parameters of the $Y(4220)$. The fit determines the mass of the $Y(4220)$ as $(4219.6 \pm 3.3 \pm$ $5.1) \mathrm{MeV} / c^{2}$ and the width is $(56.0 \pm 3.6 \pm 6.9) \mathrm{MeV}$. The coupling of this state to the lepton pair is also extracted.

\section{The $X(3872)$}

The $X(3872)$ was observed in $B^{ \pm} \rightarrow K^{ \pm} \pi^{+} \pi^{-} J / \psi$ decays 15 years ago at the Belle experiment [37]. It was confirmed subsequently by several other experiments [38-40]. BESIII observed a new process of the $X(3872)$ production: $e^{+} e^{-} \rightarrow \gamma X(3872) \rightarrow \gamma \pi^{+} \pi^{-} J / \psi[41]$.

The $X(3872)$ signal is shown in Fig. 7 (left), the mass is measured as $(3871.9 \pm 0.7 \pm$ 0.2) $\mathrm{MeV} / \mathrm{c}^{2}$ and the width is less than $2.4 \mathrm{MeV}$ at the $90 \%$ C.L. The statistical significance of $X(3872)$ is $6.3 \sigma$. The energy-dependent cross sections (Fig. 7 (right)) can be described with the $Y(4260)$ resonance well, which supports the existence of radiative transition 

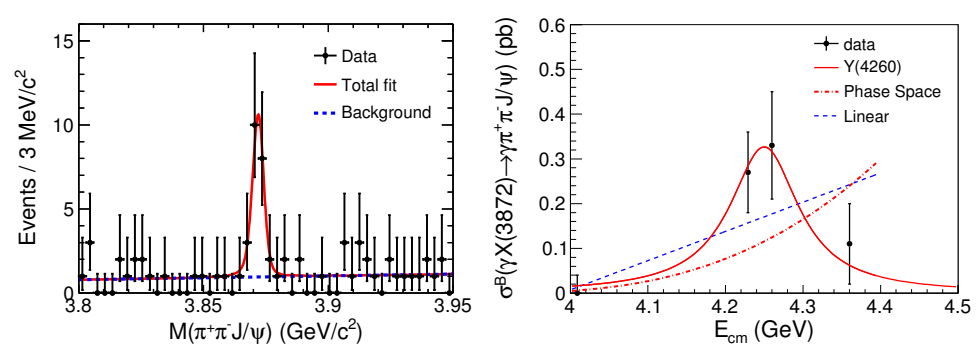

Figure 7. Fit the $M\left(\pi^{+} \pi^{-} J / \psi\right)$ distribution observed at BESIII (left panel), and fit to $\sigma^{B}\left[e^{+} e^{-} \rightarrow\right.$ $\gamma X(3872)] \times \mathcal{B}\left[X(3872) \rightarrow \pi^{+} \pi^{-} J / \psi\right]$ (right panel).

$Y(4260) \rightarrow \gamma X(3872)$. Together with the hadronic transition of the $Y(4260) \rightarrow \pi Z_{c}(3900)[6-$ $8]$, these suggest that there might be some commonality in the nature of $X(3872), Y(4260)$, and $Z_{c}(3900)$, and so the model developed to interpret any one of them should also consider the other two.

\section{Summary and Perspectives}

BESIII has achieved a lot in the study of the XYZ states. There are still some data at BESIII not analyzed and data at more energy points will be taken [42]. More analyses with these data samples will allow many improved understanding of the XYZ states, especially the $X(3872)$, $Y(4260), Z_{c}(3900)$, and $Z_{c}(4020)$. BEPCII is upgrading the maximum c.m. energy from 4.6 to $4.9 \mathrm{GeV}$ in two years, this will enable a full coverage of the $Y(4660)$ [23] and $Y(4630)$ [43] resonances, improved measurements of their properties are expected.

\section{Acknowledgments}

Many thanks to the organizers for the invitation to give this talk and for the perfectly organized conference. This work is supported in part by National Natural Science Foundation of China (NSFC) under contract Nos. 11475187, 11521505, and 11835012; the Ministry of Science and Technology of China under Contract No. 2015CB856701; Key Research Program of Frontier Sciences, CAS, Grant No. QYZDJ-SSW-SLH011; and the CAS Center for Excellence in Particle Physics (CCEPP).

\section{References}

[1] C. Patrignani et al. [Particle Data Group], Chin. Phys. C 40, 100001 (2016). M. Tanabashi et al. [Particle Data Group], Phys. Rev. D 98, 030001 (2018).

[2] A. J. Bevan et al. [BaBar and Belle Collaborations], Eur. Phys. J. C 74, 3026 (2014).

[3] For recent reviews, see F. K. Guo, C. Hanhart, U. G. Meißner, Q. Wang, Q. Zhao and B. S. Zou, Rev. Mod. Phys. 90, no. 1, 015004 (2018). H. X. Chen, W. Chen, X. Liu and S. L. Zhu, Phys. Rept. 639, 1 (2016); N. Brambilla et al., Eur. Phys. J. C 71, 1534 (2011).

[4] M. Ablikim et al. [BESIII Collaboration], Nucl. Instrum. Methods Phys. Res., Sect. A 614, 345 (2010). 
[5] More results from BESIII can be found in C. Z. Yuan, “The XYZ states revisited," Int. J.

Mod. Phys. A 33, no. 21, 1830018 (2018) [arXiv:1808.01570 [hep-ex]].

[6] M. Ablikim et al. [BESIII Collaboration], Phys. Rev. Lett. 110, 252001 (2013).

[7] Z. Q. Liu et al. [Belle Collaboration], Phys. Rev. Lett. 110, 252002 (2013).

[8] T. Xiao, S. Dobbs, A. Tomaradze and K. K. Seth, Phys. Lett. B 727, 366 (2013).

[9] M. Ablikim et al. [BESIII Collaboration], Phys. Rev. Lett. 119, 072001 (2017).

[10] M. Ablikim et al. [BESIII Collaboration], Phys. Rev. Lett. 112, 022001 (2014).

[11] M. Ablikim et al. [BESIII Collaboration], Phys. Rev. D 92, 092006 (2015).

[12] Chang-Zheng Yuan, talk at "The 9th International Workshop on Charm Physics",

May 21 to 25, 2018, Novosibirsk, Russia. https://indico.inp.nsk.su/event/10/session/2/ contribution/41/material/slides/0.pdf

[13] A. Esposito, A. L. Guerrieri and A. Pilloni, Phys. Lett. B 746, 194 (2015).

[14] G. Li, X. H. Liu and Z. Zhou, Phys. Rev. D 90, 054006 (2014).

[15] H. W. Ke, Z. T. Wei and X. Q. Li, Eur. Phys. J. C 73, 2561 (2013).

[16] L. Ma, X. H. Liu, X. Liu and S. L. Zhu, Phys. Rev. D 91, 034032 (2015).

[17] L. Ma, W. Z. Deng, X. L. Chen and S. L. Zhu, arXiv:1512.01938 [hep-ph].

[18] M. B. Voloshin, arXiv:1806.05651 [hep-ph].

[19] M. Ablikim et al. [BESIII Collaboration], Phys. Rev. Lett. 111, 242001 (2013).

[20] M. Ablikim et al. [BESIII Collaboration], Phys. Rev. Lett. 112, 132001 (2014).

[21] B. Aubert et al. [BaBar Collaboration], Phys. Rev. Lett. 95, 142001 (2005).

[22] B. Aubert et al. [BaBar Collaboration], Phys. Rev. Lett. 98, 212001 (2007).

[23] X. L. Wang et al. [Belle Collaboration], Phys. Rev. Lett. 99, 142002 (2007).

[24] M. Ablikim et al. [BESIII Collaboration], Phys. Rev. Lett. 118, 092001 (2017).

[25] J. P. Lees et al. [BaBar Collaboration], Phys. Rev. D 86, 051102(R) (2012).

[26] M. Ablikim et al. [BESIII Collaboration], Phys. Rev. Lett. 111, 242001 (2013).

[27] M. Ablikim et al. [BESIII Collaboration], Phys. Rev. Lett. 118, 092002 (2017).

[28] M. Ablikim et al. [BESIII Collaboration], Phys. Rev. Lett. 114, 092003 (2015).

[29] J. P. Lees et al. [BaBar Collaboration], Phys. Rev. D 89, 111103 (2014).

[30] X. L. Wang et al. [Belle Collaboration], Phys. Rev. D 91, 112007 (2015).

[31] M. Ablikim et al. [BESIII Collaboration], Phys. Rev. D 96, 032004 (2017).

[32] M. Ablikim et al. [BESIII Collaboration], Phys. Rev. D 93, 011102 (2016).

[33] M. Ablikim et al. [BESIII Collaboration], arXiv:1808.02847 [hep-ex], submitted to

Phys. Rev. Lett.

[34] G. Pakhlova et al. [Belle Collaboration], Phys. Rev. D 80, 091101 (2009).

[35] X. Y. Gao, C. P. Shen and C. Z. Yuan, Phys. Rev. D 95, 92007 (2017).

[36] J. Zhang, L. Yuan and R. Wang, arXiv:1805.03565 [hep-ph].

[37] S. K. Choi et al. [Belle Collaboration], Phys. Rev. Lett. 91, 262001 (2003).

[38] D. Acosta et al. [CDF Collaboration], Phys. Rev. Lett. 93, 072001 (2004).

[39] V. M. Abazov et al. [D0 Collaboration], Phys. Rev. Lett. 93, 162002 (2004).

[40] B. Aubert et al. [BaBar Collaboration], Phys. Rev. D 71, 071103 (2005).

[41] M. Ablikim et al. [BESIII Collaboration], Phys. Rev. Lett. 112, 092001 (2014).

[42] Chang-Zheng Yuan, Front. Phys. 10, 101401 (2015).

[43] G. Pakhlova et al. [Belle Collaboration], Phys. Rev. Lett. 101, 172001 (2008). 